\title{
Research on Learning Behavior of Undergraduates in E-learning Environment ${ }^{*}$
}

\author{
WenfengWang $^{1}$, YangboJiang ${ }^{2}$, FangDong $^{3}$ \\ ${ }^{1)}$ School of Information Engineering, Nanchang Institute of Technology, Nanchang 330099, China \\ ${ }^{2)}$ Network information center, Nanchang Institute of Technology, Nanchang 330099, China \\ ${ }^{3)}$ Department of Scientific Research, Nanchang Institute of Technology, Nanchang 330099, China \\ wangwf@nit.edu.cn, jyb@nit.edu.cn, dongf@nit.edu.cn
}

\begin{abstract}
Autonomy, interactivity, cooperativity, flexibility and etc., all these luxuries contribute to the popularity of online learning. More and more people tend to acquire all kinds of learning resources from the Internet. Learners could only obtain limited learning resources by wired-network-accessed computers in the past, while they can get massive diversified resources by wireless-accessed mobile phones. Therefore, it is worthy to study the learning behaviors of undergraduates in the current learning environment. In this paper, we firstly present the online learning data of undergraduates from the Nanchang Institute of Technology, and then we analyze their learning behaviors. Finally, we propose some relative strategies. Teaching practice demonstrates that they are effective to promote the quality of teaching.
\end{abstract}

Keywords-Online learning, Teaching model, Learning behavior, Integrated platform of network teaching

\section{E-learning 环境下的大学生在线学习行为特征研究}

\author{
王文丰 $^{1}$ 江阳波 $^{2}$ 董芳 $^{3}$ \\ 1) 南昌工程学院 信息工程学院, 江西 南昌 330099 \\ 2) 南昌工程学院网络信息中心, 江西 南昌 330099 \\ 3) 南昌工程学院 科学研究处, 江西 南昌 330099
}

摘 要 在高校信息化、网络化建设加速发展的环境下, E-learning 在线学习已经成为大学生学习的主要形式之一, 学生通过手机和 电脑上网获得各种学习资源。本文通过对南昌工程学院学生在线学习数据的研究, 分析了在校大学生在线学习行为, 并就改善学生在 线学习行为方式提出了相应对策。教学实践表明, E-Learning 教学方式有效提高了学生的学习积极性, 激发了学生的学习热情, 具有 良好的教学效果。

关键词 在线学习; 学习方式; 学习行为; 网络教学综合平台

1. 引言

随着互联网技术和高校信息化建设的迅猛发展, 网络 已经成为学习生活中不可或缺的一部分, 大学生利用网络 学习的时间也越来越多, 大量网络化、信息化、数字化的
学习工具被运用到教学中, 以充分利用大学生 E-learning 环 境下这一新型学习行为方式。E-learning 这种新型学习形式 是以现代网络技术为基础, 学习者利用网络提供的各种工 具实现远程学习、交流, 从而获得老师、专家, 同学的帮

“江西省教育科学 “十二五” 规划 2012 年度重点课题 “E-Learning 环境下大学生在线学习行为特征研究一以南昌工程学院为例” (编号: 12ZD073), 南昌工程学院校级教学改革研究项目资助。 
助及学习资源, 达到学习目的。E-learning 以其自主性、 交互性、协作性、实时性等特点受到大学生的喜爱, 大学 生可以根据自己的学习能力来控制学习进度, 通过交流来 增进自己学习兴趣和改进学习方法。随着 E-learning 的不 断发展和网络教学资源的不断改善, 南昌工程学院也发展 了自己的 E-learning 学习平台—网络教学综合平台。

\section{2. 南昌工程学院在线学习环境的运行现状}

\section{1 南昌工程学院 E-learning 环境结构特点}

由于高校教育教学建设快速发展, 教学方式也在不断 发展演化, 从早期的“导师式教学”演变为标准化、大规模 的“班级授课制”进而发展为互联网时代的“网络远程教学”。 E-learning 学习平台也从早期的“个性化学习平台”发展为
“系统化的学习平台”。南昌工程学院在线学习环境 (网络 教学综合平台) 是基于 Web 的教学管理系统, 采用 $\mathrm{B} / \mathrm{S}$ 结 构、教育技术学理论、计算机网络技术构建的集教学、教 学资源库管理, 教学管理于一体的在线学习平台。根据不 同的教学模式、不同教育对象特点, 在各种不同的教学环 节中为网络教育提供灵活的、可缩放的、适合于多种层面、 多种对象及多种网络环境的交互式教学平台, 实现课程学 习和教学。南昌工程学院综合教学平台包含了教师备课系 统、辅导答疑系统、作业评阅系统、远程考试系统、试题 生成系统、个人资源系统、教师和学生的个人信息系统及 其他辅助系统等相应模块, 教师通过网络教学平台上传自 己教学资料和相关视频, 学生通过网络教学平台进行课程 学习和答疑提问, 实现教学的互动。结构见图 1

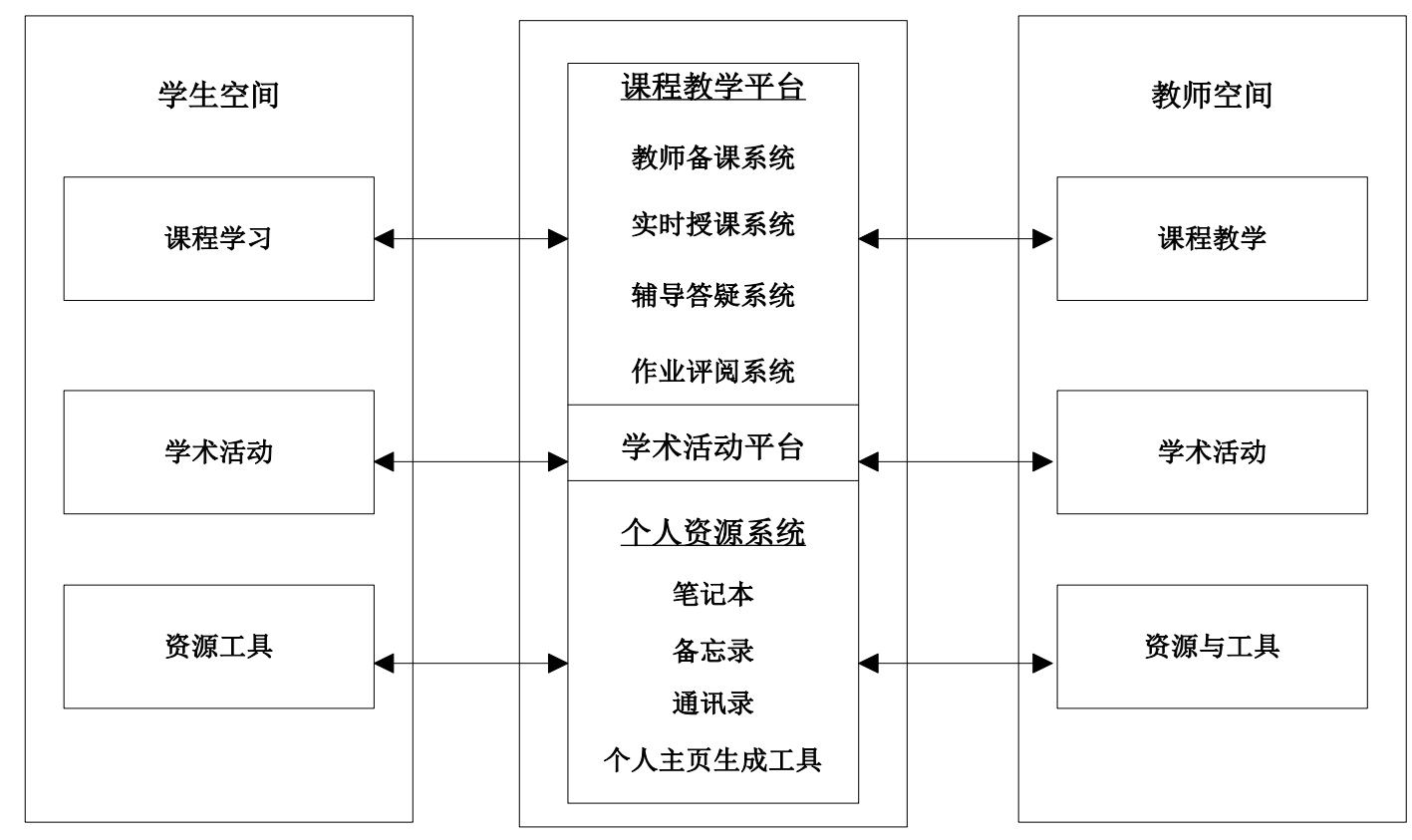

图 1 网络教学综合平台结构

\section{2 南昌工程学院 E-learning 环境资源建设}

为了解在校大学生网络学习行为现状, E-learnin 资源 建设和访问情况, 提高大学生在线学习行为主动性, 笔者 对南昌工程学院的网络教学综合平台运行数据进行整理、 统计发现曾经登录过的教师用户 867 人, 上传教学资料的 老师 619 人, 疑难问答老师 619 人, 实际网络课程 4051 门, 实际建设的课程 2156 门。学生用户数 16580 人, 学生访问 数 14324 人, 学生参与讨论数 1165 次, 课程讨论发文总数 2316, 课程布置作业数 2815。课程访问量为: 高等数学 11106 次, 计算机程序设计基础 11006 次, 毛泽东、邓小平理论 和“三个代表”重要思想概论访问量 1960 次, 组织行为学
1931 次, 审计学 2372 次, 军事理论 550 次。通过这些访问 量靠前的课程发现, 这些课程网络教学材料比较丰富, 生 动有趣, 迎合了学生的学习兴趣, 教学互动性比较强。教 师充分利用了网络教学平台开展辅助教学。

\section{3. 在校大学生 E-learning 环境下学习行为分析}

\section{1 大学生学习行为方式分析}

根据网络教学平台资源访问数据, 经统计、整理发现 $68 \%$ 的学生是基于任务学习方式, 教师添加课程作业比较多的 章节, 该章节课程访问量比较高, 并及时通过网络学习平 台提交作业, 而跟学习任务无关的课程资源访问量明显偏 
低, 甚至出现有些课程材料从来没有被访问的局面。 $22 \%$ 的学生能够基于自己的兴趣爱好, 访问其它专业领域的课 程 (如: 军事理论) 通过 E-learning 平台进行学习, $4 \%$ 的 学生比较盲目, 不能充分利用网络进行学习, $6 \%$ 的学生采 用其它方式进行学习。具体统计情况如图 2

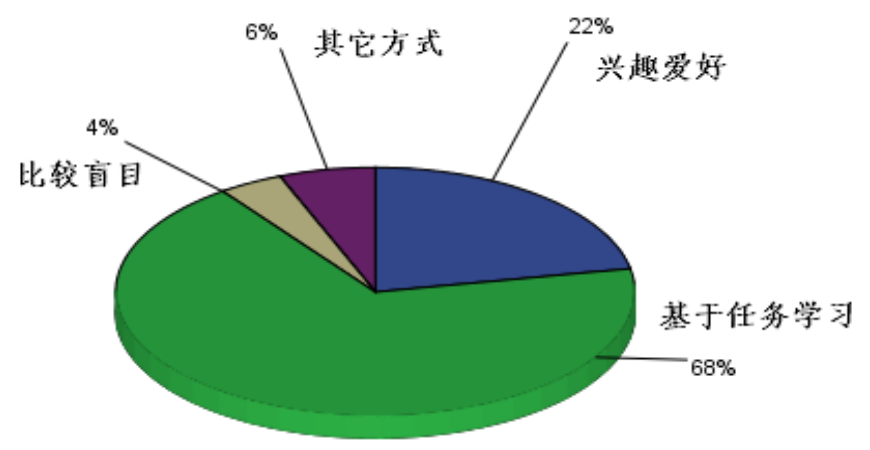

图 2 大学生学习行为方式分析

\section{2 信息资料的获取、处理}

由于课内学习的内容有限, 知识的拓展和课余信息资 料的获取显得尤为重要, 虽然目前获取信息资料的途径比 较多, 学校也建立了图书馆供学生查阅, 但网络仍然是学 生获取知识途径的最佳选择。

\section{2 .1 信息资料的获取途径}

信息网络的高速发展, 大学生上网获取信息的方式也 多样化, 通过对在校大学生“网络接入方式”问卷调查, 发 出问卷 160 份, 实际收回问卷 160 份, 有效问卷 $100 \%$, 通 过调查得知, 有线网络上网的台式机占 $5 \%$, 笔记本占 $92.5 \%$, 无线网络上网的手机占 $98 \%$, 通过 ipad 上网的占 $6.8 \%$, 笔记本占 $5 \%$, 台式机占 $2.5 \%$ 。具体情况如表 1

表 1 大学生网络接入方式

\begin{tabular}{|c|c|c|c|c|c|}
\hline 人数 & 台式机 & 笔记本 & 手机 & ipad & 其它 \\
\hline 有线网络 & 8 & 148 & 0 & 0 & 4 \\
\hline 无线网络 & 4 & 8 & 157 & 11 & 4 \\
\hline
\end{tabular}

\section{2 .2 信息资料的处理}

大学生可以通过网络浏览、搜索、查询等方式从网络 学习平台和 internet 上获取自己想要的信息, 进行学习, 在 学习过程中遇到问题时, 该如何处理? 通过表 2 可以发现, 学生在遇到学习问题时, 绝大多数学习者首先想到向百度、 google 等搜索引擎求助, 认为在这里能够更快捷的找到他
们想要的东西, 然后才是通过网络学习平台的在线答疑寻 求援助, 很少有人像课堂一样, 遇到问题请教老师和同学 或者利用其它网络交流工具进行求助。

表 2 学习问题处理方式

\begin{tabular}{|c|c|c|c|c|c|}
\hline $\begin{array}{c}\text { 问题处理 } \\
\text { 方式 }\end{array}$ & $\begin{array}{c}\text { 网络平台 } \\
\text { 在线答疑 }\end{array}$ & $\begin{array}{c}\text { 百度、 } \\
\text { google 搜 } \\
\text { 索引擎 }\end{array}$ & $\begin{array}{c}\text { 向老师、 } \\
\text { 学生求助 }\end{array}$ & $\begin{array}{c}\text { 答题库搜 } \\
\text { 索 }\end{array}$ & 其它 \\
\hline 人数 & 67 & 38 & 24 & 20 & 16 \\
\hline $\begin{array}{c}\text { 占抽样人 } \\
\text { 数百分比 }\end{array}$ & 40.6 & 23 & 14.5 & 12.1 & 9.7 \\
\hline
\end{tabular}

\section{3 大学生在线交互学习行为分析}

由于课堂时间有限, 许多问题不能深入开展, 师生、 生生之间交流不够充分, 网络能够弥补课程交流的不足, 使得交流没有时间和地域的局限, 交流方式也更加多样化, 然而在众多的网络交流方式中师生和生生之间交流方式也 有着明显的不同, 学生与老师交流主要通过 E-mail 和网络 在线答疑, 而学生之间交流往往通过 $\mathrm{QQ}$ 或微信等实时通 信工具进行点对点信息交流, 在整个交互式学习过程中有 $22 \%$ 的大学生基本上没有跟老师做过任何交流, 也有 $5 \%$ 的 同学独立独行不跟同学做任何学习交流。这说明大学生在 进行网络交互式学习时更愿意同学之间讨论, 共同解决学 习问题。

对于网络在线答疑教学活动, 根据网络教学综合平台 的教师和课程发文次数和回文次数可以发现, 有 $69 \%$ 的同 学会在活动中向老师提问, $17 \%$ 的同学多次在活动中向老 师提问, $10 \%$ 的同学虽然在线, 但参与热情不高, 一直处 于一种观望的状态, 甚至有极少数学生从没有参与过网络 答疑, 认为网络答疑都是一些聊天, 没什么作用, 属于浪 费时间的行为。具体情况如图 3 所示

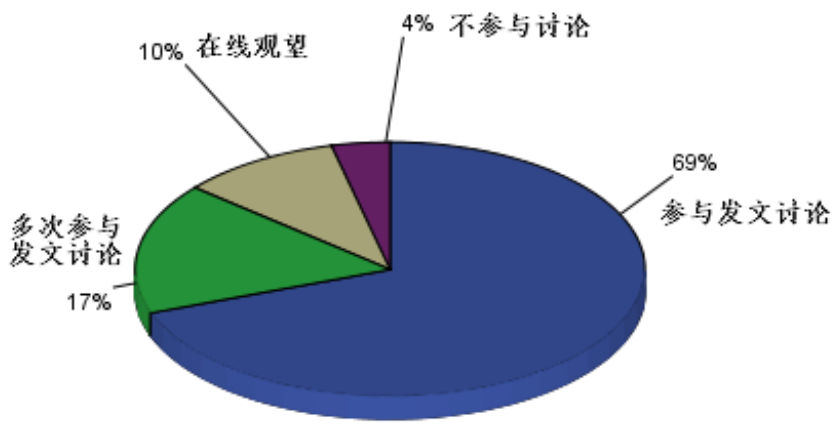

图 3 大学生在线学习行为分析 
统计分析显示回文次数是发文次数的 $96 \%$, 大部分学 生都会参与这种课题活动, 且学生的发文和回文次数占课 程讨论区次数的 $85 \%$ 。

\section{4 大学生对网络教学平台资源利用情况分析}

南昌工程学院网络教学综合平台是学生进行网络课程 学习的主要平台, 也是大学生进行在线学习过程中使用最 多的一种网络资源, 目前共有网络课程 4051 门, 精品课程 78 门, 网络辅助教学课程 87 门, 布置课程作业的 33 门, 从课程和作业的访问情况来看, 约占 $70 \%$ 的同学在进行在 线学习时, 是以“书面教材为主、网络资源为辅”查看教师 上传的视频和相关课件, 约占 $80 \%$ 的学生会登录网络教学 平台进行资料的查询, $90 \%$ 的网络课程资料被学生阅读访 问。在完成教师布置的课程作业时, 绝大多数同学都是边 学习课程内容边做习题, 来巩固自己所学知识。

南昌工程学院网络教学综合平台中包含有不同的学习 栏目, 如课件、教学视频、习题与解答、参考资料等, 在 这些栏目的相关统计中, 习题与解答以其有针对性的案例 解说受到 $40 \%$ 学生的喜爱, 这些习题与解答针对平时学习 的难点、重点进行分析, 比较容易让大学生理解接受。其 次是课件约占 $24.7 \%$ 的同学对其进行过访问, 由于这些课 件丰富记载了上课内容的一些细节, 可以方便大学生进行 课堂复习而位居次位。也有约占 $31.8 \%$ 的同学对教学视频、 参考资料等其它栏目进行过不同程度的访问。如表 3 所示

表 3 网络教学综合平台学习栏目访问统计

\begin{tabular}{|c|c|c|c|c|c|}
\hline 学习栏目 & 习题与解答 & 课件 & 教学视频 & 参考资料 & 其它 \\
\hline 访问次数 & 68 & 42 & 28 & 26 & 6 \\
\hline 占百分比 & 40 & 24.7 & 16.5 & 15.3 & 3.5 \\
\hline
\end{tabular}

\section{4. 促进大学生在线学习行为的对策}

根据上文的数据分析表明, 现在大学生大多都能适应 E-learning 在线学习这种行为方式, 并且在学习过程中能够 利用网络资源进行辅助性学习, 由于大学生在网络学习过 程中, 具有较强的目的性和针对性, 只是网络技能参差不 齐, 导致网络资源利用率不高。为了更好地促进大学生 E-learning 在线学习, 提高网络资源利用率和学校网络教学 平台的建设, 笔者提出以下几点建议:

\section{1 加强校园网信息资源环境建设}

针对学校网络平台上学习资源太少, 更新太慢的等方 面不足的问题, 首先, 学校要丰富学校网络教学综合平台
上的学习资源, 加强网络课程建设, 多开发一些学生感兴 趣的课程案例和视频资源, 着重建设一些有学校特色, 专 业型强, 对学生帮助大的课程; 其次, 为方便学生在线学 习, 放宽学生上网准入条件, 多开通一些电子阅览室, 机 房等场所, 供学生查阅网络资源。再次, 定期组织学生举 行在线学习讨论, 积极引导学生参与讨论, 让老师有效的 在讨论中跟学生进行交流, 了解学生的知识学习能力。最 后, 营造良好的网络学习氛围, 积极推进网络课堂教学, 把学校的重点课程和优秀课程做成精品网络课程在学生中 推广, 同时可以将校外名师和优秀的网络资源引入网络教 学平台, 提升网络教学资源库的建设, 提高资源利用率。 加强在学生中网络教学平台的宣传工作, 多开展网络教学 评比活动, 提高学生网络学习意识, 拓宽学习能力, 丰富 学习方式。

4.2 加强教师和学生网络技能培养, 突出教师在网络教学中 的主导地位

在网络教学活动中, 一些教师和学生网络技能不高, 学生不懂网络操作, 不能很好地利用网络进行学习, 而教 师仍然采用“黑板+课本”传统教学模式, 不熟悉使用网络进 行教学, 不知道进行网络课程建设。由于在网络教学环境 中, 教师要以引导者的身份指导学生在线学习, 制定网络 学习计划。为了更好地提升网络教学质量, 可以从两方面 着手: 一方面、在师生中普及网络基础知识, 提高他们的 网络应用水平。二、增强教师网络教学技能, 多开展网络 课堂教学, 提升师生网络教学意识和互动能力。

\section{5. 结束语}

随着网络技术的不断发展和高校信息化的建设, 网络 在线学习行为已成为大学生学习行为的重要组成部分, 网 络给大学生带来了娱乐、休闲, 也带来了丰富的资讯, 丰 富了学生交流沟通的渠道。学生在使用网络的过程中, 不 断获取网络所传递的信息, 拓宽自己的知识视野, 使得网 络成为学生获取知识的一个重要途径。如何合理的规范大 学生使用网络, 提高大学生的网络在线学习能力, 发挥网 络教育巨大优势, 是高校面临的一个重要课题, 高校应当 加快信息化建设的步伐, 积极迎接网络时代的挑战, 加强 对大学生网络学习的引导, 培养他们的网络学习素养, 丰 富学校网络资源的建设, 让学生在学校信息化建设中发挥 着积极的作用。

\section{参考文献(References)}

[1] Kashihara A., Suzuki R., Hasegawa S., et al. A Learner-centered Navigation Path Planning in Web-based Learning. Proc. of 
International Conf. on Computers in Education, 2000

[2] Wang F.S. On Analysis and Modeling of Student Browsing Behavior in Web-based Asynchronous Learning Environments. Proc. of the $1^{\text {st }}$ Int'l Conf. on Web-based Learning, 2002

[3] Hongcai Xu. Investigation and Study on the Behavior of College Students in Network Learning. Journal of E-education research (in Chinese), 2005, 6: 61-64

[4] Kebin Huang. Feng Wang. The network learning behavior study under E-learning environment. Journal of Shanxi Radio \& TV University, 2008, 2: 18-19

[5] Yi Gao.RuiMin Shen etc. Based on open E - learning platform for students behavior analysis center. Computer engineering, 2004,
30 (15) : 86-88

[6] Mario M.O., Pedro J.M.M., Carlos D.K.. Student Behavior and Interaction Patterns with an LMS as Motivation Predictors in E-Learning Settings. IEEE Tran. on Education, 2010, 53(3): 463-470

\section{作者简介:}

王文丰(1983-), 男, 湖北黄冈人, 博士, 副教授; 江阳波(1978-), 男, 江西丰城人, 工程师; 董芳(1984-), 女, 湖北黄冈人, 硕士, 讲师; 